\title{
MALAT1 affects ovarian cancer cell behavior and patient survival
}

\author{
QUNBO LIN ${ }^{1,2}$, WENCAI GUAN ${ }^{1}$, WEIMIN REN ${ }^{1,2}$, LINGYUN ZHANG $^{1,2}$, \\ JINGUO ZHANG ${ }^{1,2}$ and GUOXIONG XU ${ }^{1,2}$ \\ ${ }^{1}$ Center Laboratory, Jinshan Hospital, Fudan University, Shanghai 201508; ${ }^{2}$ Department of Oncology, \\ Shanghai Medical College, Fudan University, Shanghai 200032, P.R. China
}

Received January 9, 2018; Accepted April 12, 2018

DOI: $10.3892 /$ or.2018.6384

\begin{abstract}
Epithelial ovarian cancer (EOC) is one of the most lethal malignancies of the female reproductive organs. Increasing evidence has revealed that long non-coding RNAs (lncRNAs) participate in tumorigenesis. Metastasis associated lung adenocarcinoma transcript 1 (MALAT1) is an IncRNA and plays a role in various types of tumors. However, the function of MALAT1 on cellular behavior in EOC remains unclear. The current study explored the expression of MALAT1 in ovarian cancer tissues and in EOC cell lines. Quantitative RT-PCR analysis revealed that the expression of MALAT1 was higher in human ovarian malignant tumor tissues and EOC cells than in normal ovarian tissues and non-tumorous human ovarian surface epithelial cells, respectively. By analyzing the online database Kaplan-Meier Plotter, MALAT1 was identified to be correlated with the overall survival (OS) and progression-free survival (PFS) of patients with ovarian cancer. Furthermore, knockdown of MALAT1 by small interfering RNA (siRNA) significantly decreased EOC cell viability, migration, and invasion. Finally, dual-luciferase reporter assays demonstrated that MALAT1 interacted with miR-143-3p, a miRNA that plays a role in EOC as demonstrated in our previous study. Inhibition of MALAT1 resulted in an increase of miR-143-3p expression, leading to a decrease of CMPK protein expression. In conclusion, our results indicated that MALAT1 was overexpressed in EOC. Silencing of MALAT1 decreased EOC cell viability and inhibited EOC cell migration and invasion. These data revealed that MALAT1 may serve as a new therapeutic target of human EOC.
\end{abstract}

\section{Introduction}

Ovarian cancer (OC) is one of the most lethal malignancies of the female reproductive organs, in the world. The estimated number of new cases of OC was 22,440 and the

Correspondence to: Professor Guoxiong Xu, Center Laboratory, Jinshan Hospital, Fudan University, 1508 Longhang Road, Shanghai 201508, P.R. China

E-mail: guoxiong.xu@fudan.edu.cn

Key words: long non-coding RNA, miR-143-3p, overall survival, progression-free survival, therapeutics, tumorigenesis estimated mortality was 14,080 , accounting for $~ 5 \%$ of the 282,500 cancer-related deaths of females in the United States in 2017 (1). The most frequent type of OC is epithelial ovarian cancer (EOC), which accounts for $~ 85 \%$ of total OCs (2). In spite of developments in surgery, chemotherapy, and radiotherapy in the past decades, the overall survival (OS) rate of patients with EOC at the late stage is a consistently poor and unfavorable prognosis $(3,4)$. It is also characterized by a high probability of drug resistance, leading to treatment failure and death in the majority of patients with distant metastasis (5). Nevertheless, the underlying molecular mechanisms for tumorigenesis, tumor progression, metastasis, and chemoresistance remain unclear. Therefore, it is necessary to acquire a better understanding of the targeted molecules involved in EOC and to find new therapeutic strategies for effective and sensitive intervention of EOC.

Long non-coding RNAs (lncRNAs), which are initially regarded as transcriptional junk, are functionally classified as transcripts over 200 nucleotides in length lacking evident protein-coding capacity (6-8). Previous studies have indicated that lncRNAs participate in different aspects of tumor development, including tumorigenesis, tumor progression, and metastasis $(9,10)$. For instance, the lncRNA HOTAIR was revealed to be upregulated in breast cancer tissues and cell lines and was closely correlated with the survival and metastasis of patients (11). The lncRNA lncARSR is exosome-transmitted in renal cancer and was revealed to have a function on chemoresistance in patients with sunitinib treatment (12). The novel lncRNA UCC was found to be increased in colorectal cancer and could regulate cell growth, invasion, and tumor progression (13). The lncRNA metastasis associated lung adenocarcinoma transcript 1 (MALAT1), first named in 2003, was revealed to be associated with the metastasis of patients with non-small cell lung cancer (NSCLC) (14).

Our previous analysis revealed the clinical value of MALAT1 (15) which may potentially be applied as a new prognostic marker. It has been revealed that MALAT1 is involved in the development of various cancers, including lung, renal, hepatic, bladder, pancreatic, gastric, colorectal, brain and breast cancers (16-24). Recently, several studies indicated that MALAT1 is associated with metastasis of patients with EOC $(25,26)$. However, the effect of MALAT1 on cellular behavior in OC and the overall survival (OS) of patients with OC remain unclear. The present study aimed to examine MALAT1 expression in human EOC tissues and EOC cell 
lines and to analyze MALAT1 expression associated with the OS and progression-free survival (PFS) of patients with OC. Finally, a loss-of-function approach was used to explore the effect of MALAT1 on cellular behaviors in EOC cells.

\section{Materials and methods}

Cell line and cultivation. Human EOC cells SK-OV-3, OVCAR-3, CAOV-3 and ES-2 were purchased from the American Type Culture Collection (ATCC; Manassas, VA, USA) and A2780 was obtained from the European Collection of Authenticated Cell Cultures (ECACC, Salisbury, UK). Human non-tumorous ovarian surface epithelial cells (HOSEpiC) were obtained from Guangzhou Jennio Biotech Co., Ltd. (Guangzhou, China). SK-OV-3 and CAOV-3 cells were cultured in DMEM (Corning Life Sciences, Manassas, VA, USA). A2780, OVCAR-3 and HOSEpiC cells were respectively cultured in RPMI-1640 media (Corning Life Sciences). ES-2 cells were cultured in McCoy's 5A medium (Sigma-Aldrich; Merck KGaA, Darmstadt, Germany). All media were supplemented with $10 \%$ fetal bovine serum (FBS; Gibco; Invitrogen; Thermo Fisher Scientific, Inc., Waltham, MA, USA) and were replaced with fresh medium every three days.

Clinical specimens. All tissue samples $(n=32)$ were derived from hospitalized patients between June 2012 to October 2016 at Jinshan Hospital, Fudan University. None of the patients with OC had received chemotherapy or radiotherapy prior to surgery. Control ovarian tissues were obtained from 12 patients with non-tumorous ovaries. The tumor and normal tissue specimens were frozen in liquid nitrogen after collection and stored at $-80^{\circ} \mathrm{C}$ until use. The present study was approved by the Ethics Committee of Jinshan Hospital and informed consent was obtained from each patient.

Bioinformatics analysis. The Kaplan-Meier Plotter database, an online bioinformatics tool (www.kmplot.com), is available to evaluate the effect of genes on survival information in OC (27). Before starting the use of the tool, the samples from patients were filtered by stage, histology, grade, and treatment elements containing debulking status and applied chemotherapy. To assess the clinical value of MALAT1, patients with OC were selected for the calculation of OS and PFS and were divided into two groups using the median, a group with low expression of MALAT1 and a group with high expression of MALAT1. The Kaplan-Meier survival curve was plotted. The hazard ratio (HR) with $95 \%$ confidence intervals (CIs) and the log-rank P-value were calculated. Online software LncBase Predicted v.2 was used to predict a candidate of miRNA that interacted with MALAT1 (http://carolina.imis.athena-innovation.gr/diana_ tools/web/index.php?r=lncbasev2\%2Findex). Online software miRWalk 2.0 database was used to find a potential target of hsa-miR-143-3p (http://zmf.umm.uni-heidelberg.de/apps/zmf/ mirwalk2/index.html) (28).

Small interfering RNA (siRNA) transfection. The MALAT1siRNA (siMALAT1) and negative control-siRNA (siNC) were obtained from RiboBio Co., Ltd. (Guangzhou, China). The sequence of siMALAT1 was 5'-GCAAATGAAAGCTACC AAT-3'. Briefly, cells were seeded in a 6-well plate at a density of
$2 \times 10^{5}$ (OVCAR-3) or $1.5 \times 10^{5}$ (SK-OV-3) cells/well. After culture for $24 \mathrm{~h}$, the cells were transfected with siRNA using an X-treme GENE Transfection Reagent (Roche Applied Science, Indianapolis, IN, USA) according to the protocol recommended by the manufacturer. The cells were then collected for subsequent experiments. The knockdown efficiency of siMALAT1 was confirmed by qRT-PCR analysis.

RNA isolation and quantitative real-time PCR. Total RNA from tissues and cells was extracted using an Axygen Bioscience kit (Suzhou, China) according to the manufacturer's protocol. The cDNA was synthesized using a Transcriptor First Strand cDNA Synthesis kit (Roche Applied Science). The reaction conditions of reverse transcription were: $25^{\circ} \mathrm{C}$ for $10 \mathrm{~min}, 50^{\circ} \mathrm{C}$ for $60 \mathrm{~min}$, $85^{\circ} \mathrm{C}$ for $5 \mathrm{~min}$, and $4^{\circ} \mathrm{C}$ for $70 \mathrm{~min}$. The qPCR experiments were conducted using a SYBR-Green Master kit (Roche Applied Science). Glyceraldehyde-3-phosphate dehydrogenase (GAPDH), $18 \mathrm{~S}$ and U6 served as a control for cells, tissues, and miRNAs, respectively. The primers were: MALAT1 forward, 5'-GTG TGCCAATGTTTCGTTTG-3' and reverse, 5'-AGGAGAAAG TGCCATGGTTG-3'; hsa-miR-143-3p forward, 5'-CTGAGA TGAAGCACTGTAGCTC-3' and reverse, 5'-GTGCAGGGT CCGAGGT-3'; GAPDH forward, 5'-GCACCGTCAAGGCTG AGAAC-3' and reverse, 5'-TGGTGAAGACGCCAGTGGA-3'; 18S forward, 5'-GACTCTGGCATGCTAACTAG-3' and reverse, 5'-GACATCTAAGGGCATCACAG-3'; and U6 forward, 5'-CTC GCTTCGGCAGCACA-3' and reverse, 5'-AACGCTTCACGA ATTTGCGT-3'. The expression of the target gene was analyzed by threshold cycle $(\mathrm{Ct}) 2^{-\Delta \Delta \mathrm{Ct}}$ method obtained from Sequence Detection Software v1.4 (7300 Real-Time PCR System; Applied Biosystems; Thermo Fisher Scientific, Inc.). The assay was performed at least three times.

Cell viability assessment. In brief, cells were cultured in 96 -well plates at a density of $6 \times 10^{3}$ cells/100 $\mu \mathrm{l}$ medium/well overnight. After transfection and culture for 0,24 and $48 \mathrm{~h}$, the viability of cells was assessed using Cell Counting Kit- 8 (CCK-8; Dojindo Molecular Technologies, Inc., Kumamoto, Japan) according to the manufacturer's protocol. The optical density (OD) values at $450 \mathrm{~nm}$ were detected using a plate reader (Epoch; BioTek Instruments, Inc., Winooski, VT, USA). At least three independent experiments were conducted.

Cell migration and invasion assays. The cell migration and invasion capacities were evaluated by Transwell assays. Briefly, a Transwell chamber with a membrane of polycarbonate (6.5 $\mathrm{mm}$ in diameter with $8-\mu \mathrm{m}$ pores; Corning Life Sciences) was placed into a well of a 24 -well plate. In the lower chamber, $700 \mu \mathrm{l}$ suitable medium containing $10 \%$ FBS was added. For the migration assay, cells were seeded in the upper chamber at a concentration of $\sim 6 \times 10^{4} / 100 \mu \mathrm{l}$ with serum-free culture medium. For the invasion assay, cells were seeded in the upper chamber with a Matrigel-coated membrane (BD Biosciences, Bedford, MA, USA) at a concentration of $\sim 8 \times 10^{4}$ cells in a $100 \mu \mathrm{l}$ volume of serum-free culture medium. After incubation for $48 \mathrm{~h}$, the non-migrated or non-invaded cells on the upper chamber were carefully removed with a cotton swab and washed with phosphate-buffered saline (PBS). Migrated or invaded cells on the reversed membrane were fixed with $4 \%$ paraformaldehyde for $15 \mathrm{~min}$, stained with crystal violet 
(Sigma-Aldrich; Merck KGaA) for 30 min, photographed, and finally counted in three random fields under a light microscope (BX43; Olympus, Tokyo, Japan) at an x200 magnification. Experiments were conducted three times.

Dual-luciferase reporter assay. 293T cells were cultured in a 24 -well plate and 70-80\% confluency was reached prior to the experiment. Cells were co-transfected with $0.4 \mu \mathrm{g}$ luciferase reporter vector (pmirGLO-MALAT1-wt or pmirGLO-MALAT1-mut) and $50 \mathrm{nM}$ miRNA (miR-143-3p mimics or miR-negative control (miR-Ctrl; RiboBio Co., Ltd.) using the X-tremeGENE Transfection Reagent (Roche Applied Science) and cultured for $24 \mathrm{~h}$. Firefly and Renilla luciferase activities were detected using Luc-Pair ${ }^{\mathrm{TM}}$ Duo-Luciferase Assay Kit 2.0 (GeneCopoeia, Inc., Rockville, MD, USA) following co-transfection according to the instructions recommended by the manufacturer. The relative firefly luciferase activity was corrected in accordance with the Renilla luciferase activity.

Western blot analysis. SK-OV-3 and OVCAR-3 cells were lysed in SDS buffer with a phosphatase inhibitor (Nanjing KeyGen Biotech Co., Ltd., Nanjing, China). The protein concentration was determined using a BCA Protein Assay kit (Thermo Fisher Scientific, Inc.). After separation on SDS-PAGE, total proteins were transferred to a PVDF membrane (EMD Millipore, Billerica, MA, USA) and incubated with either mouse anti-CMPK (cytidine monophosphate kinase; Cell Signaling Technology, Inc., Danvers, MA, USA) or rabbit anti-GAPDH (Abcam, Cambridge, UK) primary antibody at $4^{\circ} \mathrm{C}$ overnight. After incubation with horseradish peroxidase (HRP)-conjugated goat anti-mouse or anti-rabbit $\mathrm{IgG}$ (Cell Signaling Technology) for $1 \mathrm{~h}$ at room temperature, the signals were detected using Tanon- 4500 Gel Imaging System (Tanon Science and Technology Co., Ltd., Shanghai, China) with an Immobilon ${ }^{\mathrm{TM}}$ Western Chemiluminescent HRP Substrate (EMD Millipore).

Statistical analysis. SPSS 18.0 software (SPSS Inc., Chicago, IL, USA) was used to analyze the collected data. For comparison between two groups, a Student's t-test was used. The survival curve was evaluated with a log-rank test. All data are displayed as the mean \pm the standard error of the mean (SEM) from three independent experiments. A P-value $<0.05$ was considered to indicate a statistically significant difference.

\section{Results}

MALAT1 is upregulated in human epithelial ovarian cancer tissues and cell lines. In order to identify functional MALAT1 relevant to the progression of ovarian tumors, we performed qRT-PCR to evaluate the expression of MALAT1 in human ovarian normal tissues $(\mathrm{n}=12)$, benign tumors $(\mathrm{n}=8)$, and malignant tumors $(\mathrm{n}=12$, serous adenocarcinoma). The results revealed that the expression level of MALAT1 was significantly higher in ovarian malignant tumors than normal ovarian tissues and ovarian benign tumors $(\mathrm{P}<0.01)$ (Fig. 1A). In addition, the expression of MALAT1 between diverse OC cell lines and normal ovarian HOSEpiC cells was detected by qRT-PCR The expression level of MALAT1 was high in EOC cell lines SK-OV-3, OVCAR-3, CAOV-3 and A2780 cells compared to
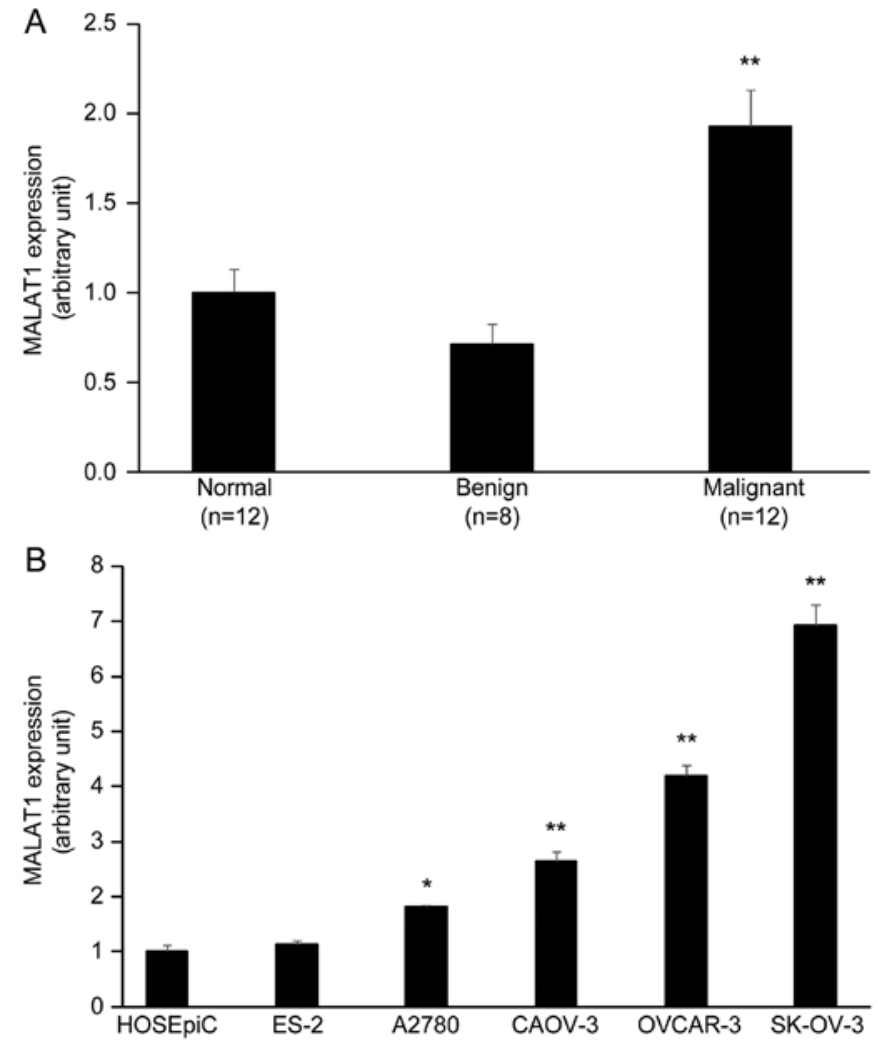

Figure 1. MALAT1 expression in human ovarian cancer. (A) Relative expression of MALAT1 was detected by qRT-PCR in the normal ovarian tissues $(n=12)$, ovarian benign $(n=8)$, and malignant $(n=12)$ tumors. (B) The expression of MALAT1 was detected by qRT-PCR in diverse cell lines, including HOSEpiC, ES-2, A2780, CAOV3, SK-OV-3 and OVCAR-3 cells ( $\mathrm{n}=3$ repeats for each cell line). The results are presented as the mean \pm SEM. ${ }^{*} \mathrm{P}<0.05$; ${ }^{* *} \mathrm{P}<0.01$. MALAT1, metastasis associated lung adenocarcinoma transcript 1 .

normal ovarian HOSEpiC cells and ovarian clear cell carcinoma ES-2 cells $(\mathrm{P}<0.05)$ (Fig. 1B).

MALAT1 is involved in the development of ovarian cancer. Based on the Kaplan-Meier Plotter online database, we further analyzed the effect of MALAT1 on the OS and PFS of patients with OC. The survival plots revealed that the expression levels of MALAT1 were correlated with OS and PFS. The patients with high MALAT1 expression had low OS as shown in Fig. 2A (Affymetrix ID: 226675_s_at) and Fig. 2B (Affymetrix ID: 224567_x_at) and PFS as shown in Fig. 2C (Affymetrix ID: 226675_s_at) and Fig. 2D (Affymetrix ID: 224567_x_at) (all $\mathrm{P}<0.05)$.

Knockdown of MALAT1 decreases ovarian cancer cell viability. In order to investigate the potential function of MALAT1 on the biological behaviors of OC cells, we conducted a loss-of-function assay. Knockdown of MALAT1 by MALAT1-siRNA (siMALAT1) was confirmed by qRT-PCT in OVCAR-3 and SK-OV-3 cells (Fig. 3A and B). Cells transfected with siMALAT1 had a low expression of MALAT1 compared with cells transfected with negative control-siRNA (siNC) and blank control (Blank). Next, we assessed cell viability using a CCK-8 assay. We found that the knockdown of MALAT1 significantly inhibited OC cell viability after $48 \mathrm{~h}$ of transfection with siMALAT1 (Fig. 3C and D). 
A

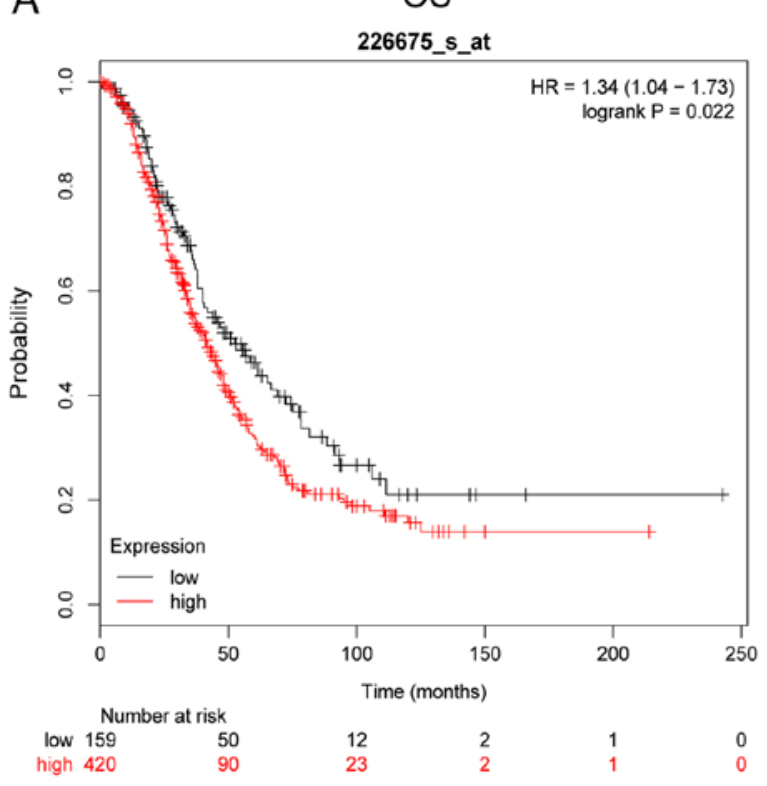

C

PFS

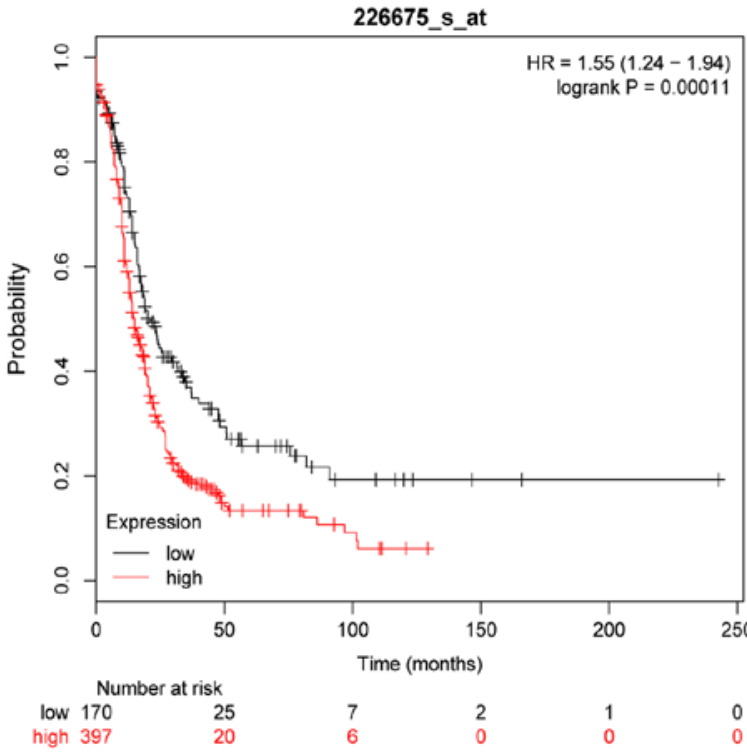

B

OS

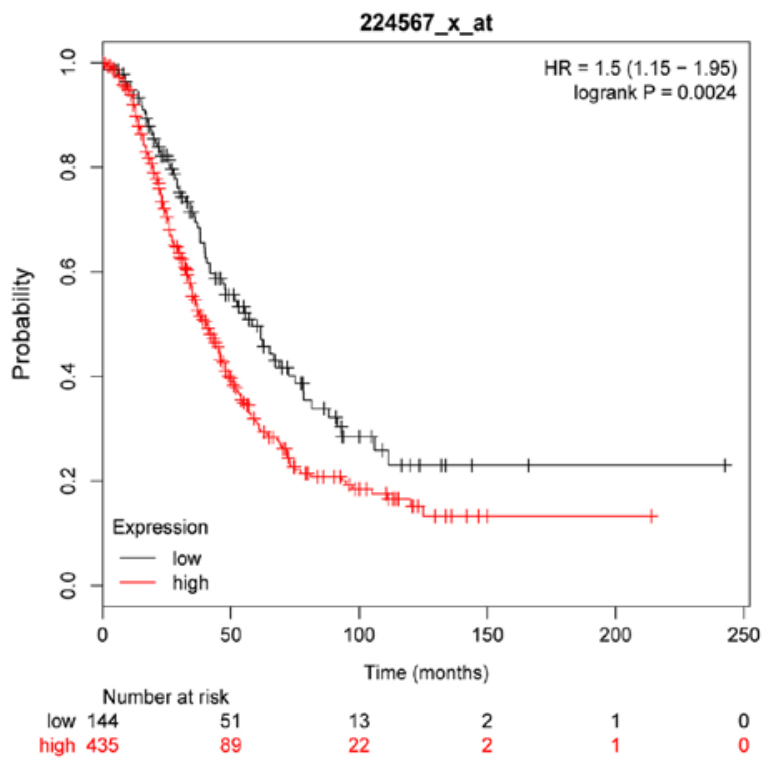

D

PFS

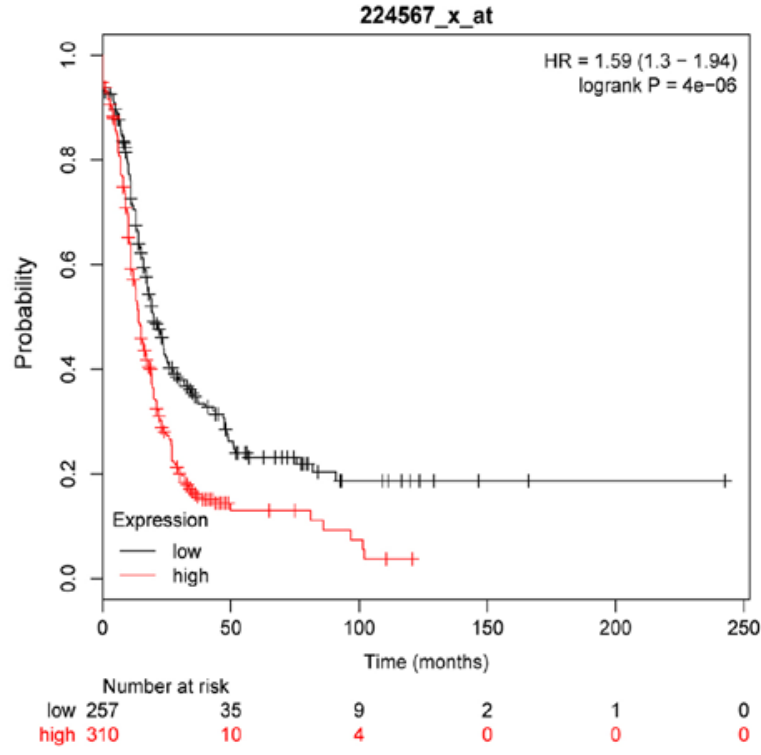

Figure 2. Survival plots. (A and B) Kaplan-Meier OS curves and (C and D) PFS curves are presented. All patients were divided into two groups based on the expression of MALAT1: A high expression group and a low expression group. (A) OS curves derived from a microarray (Affymetrix ID: 226675_s_at). (B) OS curves derived from a microarray (Affymetrix ID: 224567_x_at). (C) PFS curves derived from a microarray (Affymetrix ID: 226675_s_at). (D) PFS curves derived from a microarray (Affymetrix ID: 224567_x_at). The HR with 95\% CIs, and log-rank P for OS and PFS were calculated, respectively. OS, overall survival; PFS, progression-free survival; MALAT1, metastasis associated lung adenocarcinoma transcript 1; HR, hazard ratio; CIs, confidence intervals.

Downregulation of MALAT1 expression suppresses ovarian cancer cell migration and invasion. Next, we investigated the effect of MALAT1 knockdown on OC cell migration and invasion. Using Transwell migration assays, we determined that the number of migrated cells of OVCAR-3 (Fig. 4A and B) and SK-OV-3 (Fig. 4C and D) was significantly decreased after MALAT1-siRNA transfection (siMALAT1) for $48 \mathrm{~h}$ compared with non-transfected cells (Blank) and negative control-siRNA (siNC) transfected cells. Furthermore, the knockdown of MALAT1 also significantly decreased the number of invaded cells of OVCAR-3 (Fig. 5A and B) and SK-OV-3 (Fig. 5C and D).
MALAT1 interacts with miR-143-3p. One of the functions of lncRNAs is to regulate RNA expression. The regulatory mechanism of lncRNAs on miRNAs is that lncRNAs can act as sponges to influence the function of miRNAs (29). Using online software LncBase Predicted v.2, we predicted a candidate of miRNA hsa-miR-143-3p that is a potential target of MALAT1. Using the loss-of-function approach, we demonstrated for the first time that MALAT1 expression was correlated with miR-143-3p expression. The expression of miR-143-3p was significantly increased in OVACR-3 (Fig. 6A) and SK-OV-3 (Fig. 6B) cells after MALAT1 knockdown (siMALAT1) for $48 \mathrm{~h}$ compared with the controls (Blank 

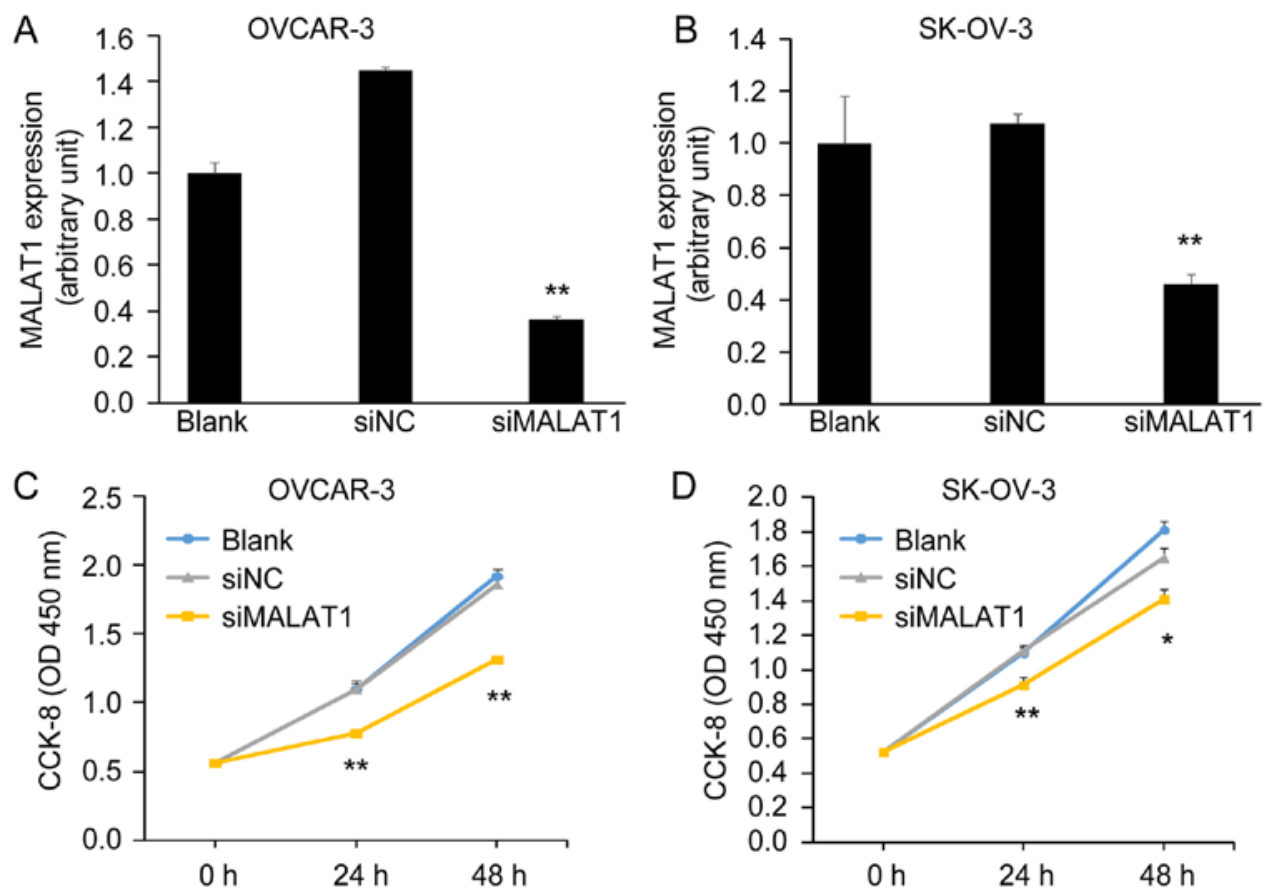

Figure 3. Effect of MALAT1-siRNA on ovarian cancer cell viability. (A and C) OVCAR-3 and (B and D) SK-OV-3 cells were transiently transfected with MALAT1-siRNA (siMALAT1) or negative control-siRNA (siNC). Non-transfected cells were used as a blank control (Blank). (A and B) MALAT1 expression was detected by qRT-PCR at $48 \mathrm{~h}$ post-transfection. (C and D) A time-course study revealing EOC cell viability as detected by CCK-8 assay. The results are presented as the mean \pm SEM. ${ }^{*} \mathrm{P}<0.05 ;{ }^{* *} \mathrm{P}<0.01 ; \mathrm{n}=3$ independent experiments. MALAT1, metastasis associated lung adenocarcinoma transcript 1 ; EOC, epithelial ovarian cancer.

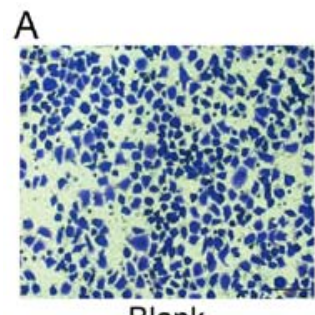
Blank

C

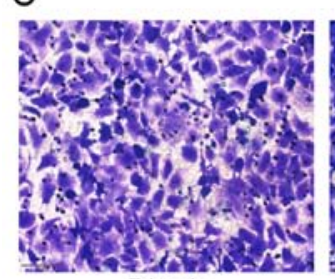

Blank
OVCAR-3

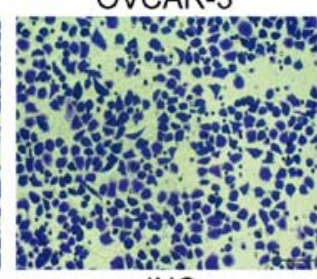

siNC

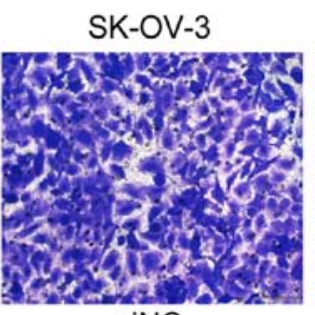

siNC
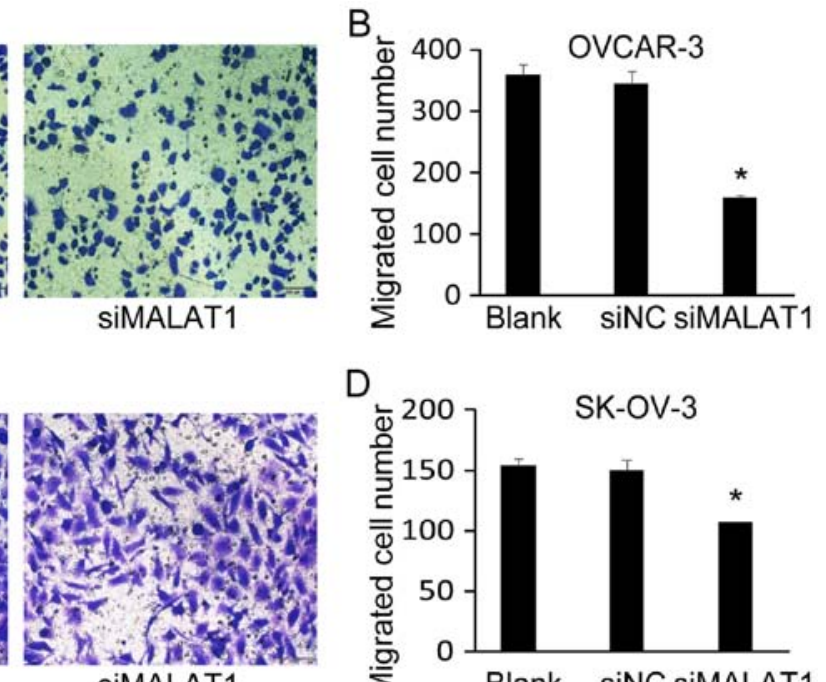

SIMALAT1

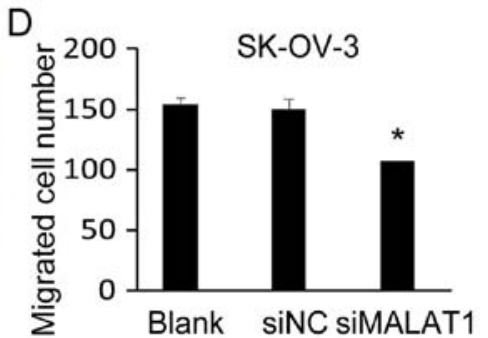

Figure 4. Effect of MALAT1-siRNA on cell migration. (A and B) OVCAR-3 and (C and D) SK-OV-3 cell migration was performed using Transwell migration assays. The cells were transfected with MALAT1-siRNA (siMALAT1) or negative control-siRNA (siNC). Cells without transfection were used as a blank control (Blank). The effective migrating cells were counted and photographed. Original amplification, x200; scale bar, $100 \mu \mathrm{m}$. The histogram displays the quantitative analysis of migrated cells. The results are presented as the mean \pm SEM. ${ }^{*} \mathrm{P}<0.05 ; \mathrm{n}=3$ independent experiments. MALAT1, metastasis associated lung adenocarcinoma transcript 1 .

and siNC) $(\mathrm{P}<0.05)$ as detected by qRT-PCR. Next, we used a dual-luciferase reporter assay to confirm the interaction between MALAT1 and miR-143-3p. Three sequences are shown in Fig. 6C: hsa-miR-143-3p, a wild-type MALAT1 containing a miR-143-3p binding site (position at 3990-3997 of MALAT1), and a mutated MALAT1 in which the binding site was changed. The Dual-Luciferase reporter assay confirmed that wild-type MALT1, not mutated MALAT1, bound to miR-143-3p in 293T cells after $72 \mathrm{~h}$ of co-transfection (Fig. 6D). These data revealed that MALAT1 could directly bind to miR-143-3p. Using miRWalk 2.0 database, we found that miR-143-3p potentially targets CMPK, a molecule previously demonstrated to play a role in EOC (30). Western blotting revealed that treating OVCAR-3 and SK-OV-3 cells with miR-143-3p mimics significantly decreased CMPK protein expression (Fig. 6E and F). 
A
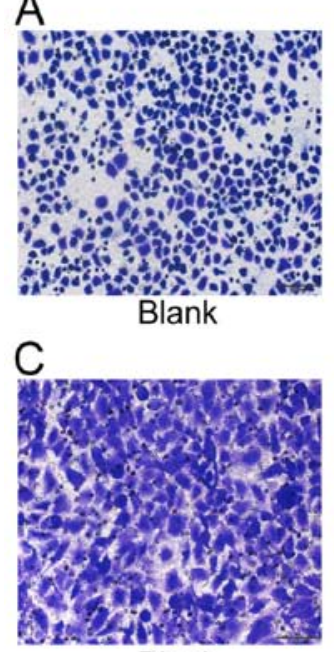

Blank

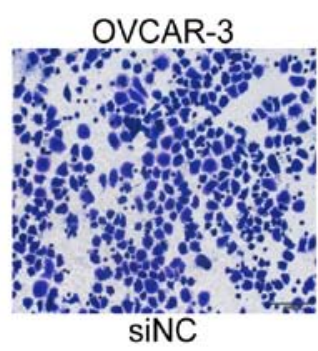

SK-OV-3

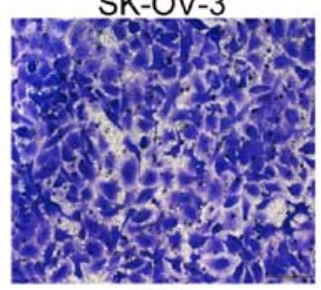

siNC

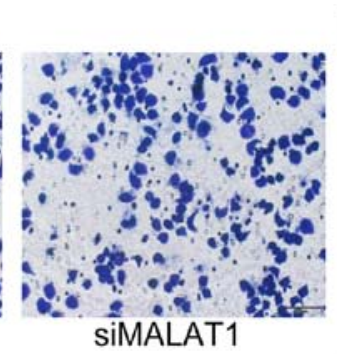

B
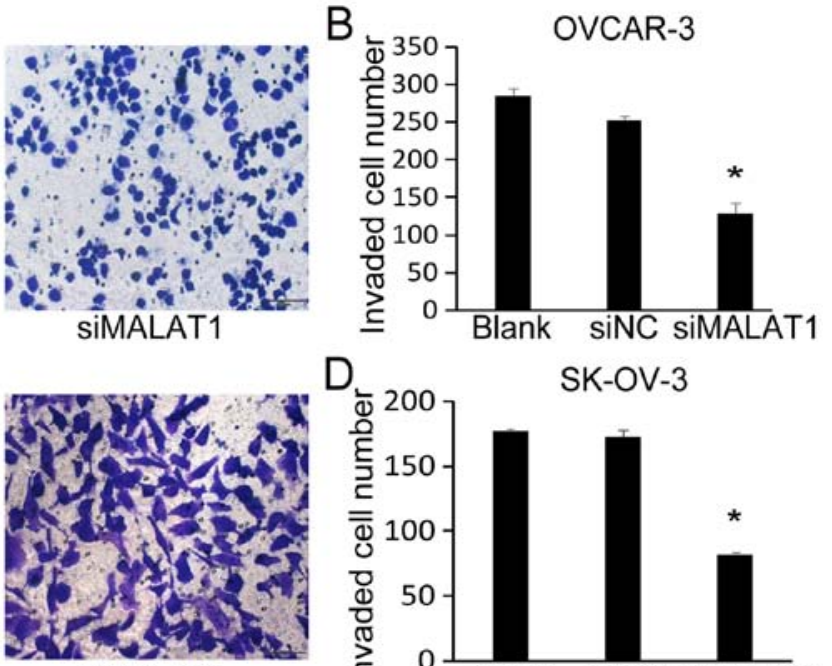

SIMALAT1

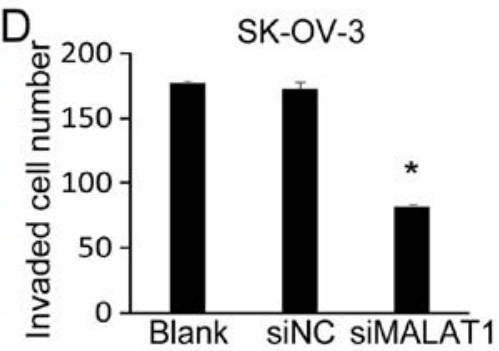

Figure 5. Effect of MALAT1-siRNA on cell invasion. (A and B) OVCAR-3 and (C and D) SK-OV-3 cell invasion was performed using Transwell invasion assays. The cells were transfected with MALAT1-siRNA (siMALAT1) or negative control-siRNA (siNC). Cells without transfection were used as a blank control (Blank). The effective invaded cells were counted and photographed. Original amplification, x200; scale bar, $100 \mu \mathrm{m}$. The histogram displays the quantitative analysis of invaded cells. The results are presented as the mean $\pm \mathrm{SEM}$. ${ }^{*} \mathrm{P}<0.05 ; \mathrm{n}=3$ independent experiments. MALAT1, metastasis associated lung adenocarcinoma transcript 1.

\section{Discussion}

The present study demonstrated that MALAT1 was overexpressed in human ovarian malignant tissues and influenced the survival of patients with OC. MALAT1, an IncRNA, plays a role in the regulation of miRNAs, which further affect downstream gene regulation.

It has been revealed that MALAT1 is upregulated in several malignant tumors such as breast $(24,31)$, bladder (32), pancreatic $(20,33)$ and colorectal (22) cancers. Our present data revealed a similar result. MALAT1 was overexpressed in ovarian malignant tumors compared with benign tumors and normal ovarian tissue. Moreover, we also observed the high expression of MALAT1 in several EOC cell lines compared with non-tumorous human ovarian surface epithelial cells (HOSEpiC). These data indicated that MALAT1 plays a role in EOC.

Through an online bioinformatics tool, we determined that MALAT1 could be a predictive biomarker of the survival of patients with OC. The available survival curves from the Kaplan-Meier Plotter database were analyzed and they revealed that a high expression of MALAT1 was associated with poor OS and PFS in OC patients. Previous studies from us and other research groups have revealed the clinical significance of MALAT1 $(15,32)$. The overexpression of MALAT1 was correlated with a decrease of disease-specific survival of patients with breast cancer (24). Elevated plasma MALAT1 was associated with distant metastasis in patients with EOC (25). However, the possible regulation mechanism remains unclear.

Our functional assays revealed that the knockdown of MALAT1 significantly inhibited OC cell viability, migration, and invasion. Similar results have been reported by other research groups. For instance, the inhibition of MALAT1 expression decreased OC cell proliferation, migration and invasion $(34,35)$. MALAT1 induced EOC cell proliferation via the PI3K/Akt signaling pathway (26). These data indicated that MALAT1 may play a role in OC cell behavior.

MALAT1 can act as a regulator of the expression of other RNAs such as miRNAs and forms a molecular interaction network in different types of cancer $(36,37)$. Recent studies have revealed that MALAT1 can target various miRNAs, which partly explains the mechanism of MALAT1 which plays a role in disease processes $(38,39)$. For instance, miR-200s was revealed to be sponged by MALAT1 in clear cell kidney carcinoma (40). miR-206 was determined to be negatively regulated by MALAT1 in gallbladder cancer (41). Using online software, we identified miR-143-3p as a possible target of MALAT1. Our Dual-Luciferase reporter assay demonstrated the interaction between MALAT1 and miR-143-3p, indicating that MALAT1 is capable of functioning as a molecular sponge to adsorb miR-143-3p and subsequently regulate OC cell behaviors. Indeed, inhibition of MALAT1 resulted in an increase of miR-143-3p. Due to the size of MALAT1 which is over $8,000 \mathrm{nt}$ in length (14), we were not able to obtain a full-length clone. Currently, we are unable to do a gain-of-function assay.

Several recent studies have revealed that miRNAs, a class of non-coding RNAs $\sim 22 \mathrm{nt}$ in length, are subject to the regulation of various biological processes as part of an integrated pathophysiological response to various stimuli $(42,43)$. It has been revealed that miR-143-3p could play a role in tumorigenesis and function as a tumor suppressor gene in breast cancer and esophageal squamous cell carcinoma $(44,45)$. We recently revealed that CMPK plays a role in ovarian tumorigenesis (30). Based on the bioinformatics analysis and our further experiments, we demonstrated that CMPK was one of the targets of miR-143-3p. It may be considered that miR-143-3p is a tumor-inhibitory factor by targeting CMPK in OC. These results ascertained that MALAT1 negatively regulated miR-143-3p via a sponge-like function, and in turn, 
A

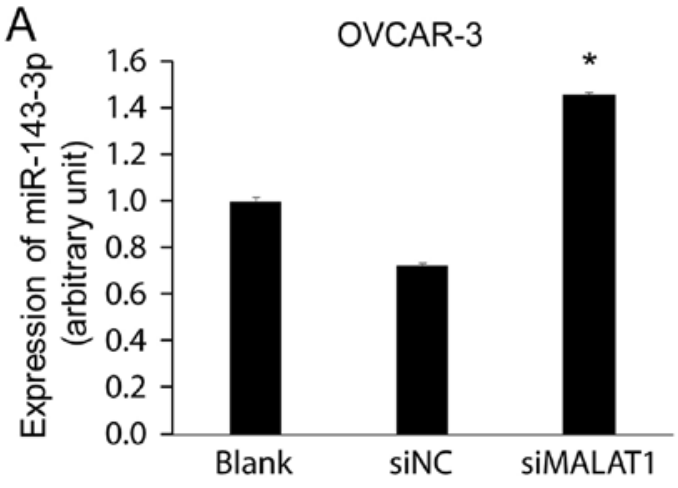

B

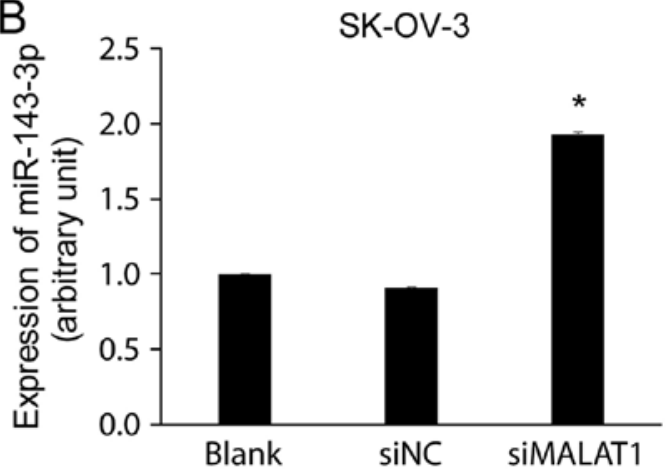

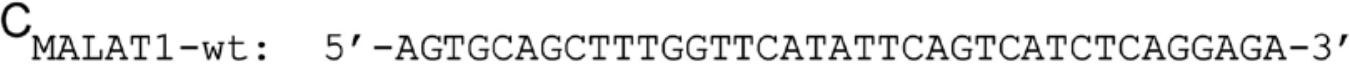 \\ hsa-miR-143-3p:

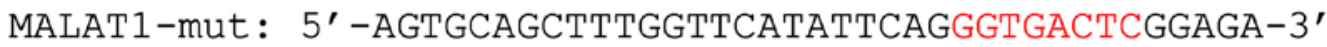

D

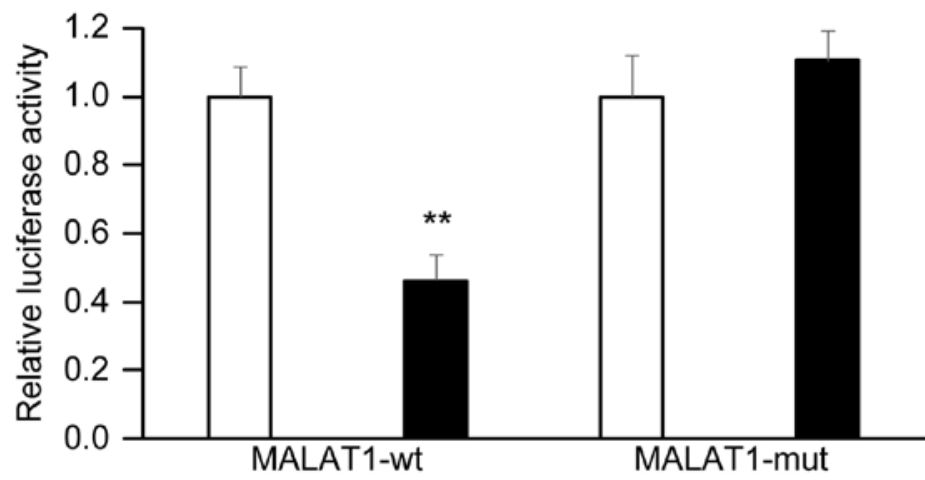

miR-Ctrl

$\operatorname{miR}-143-3 p$

E

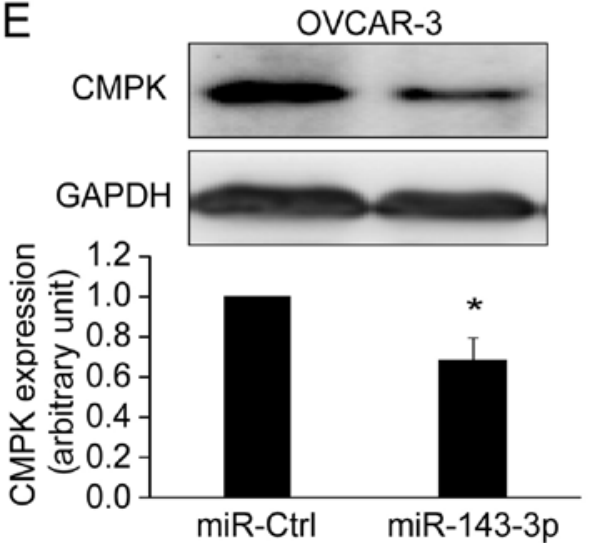

F

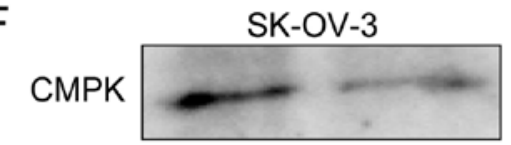

GAPDH

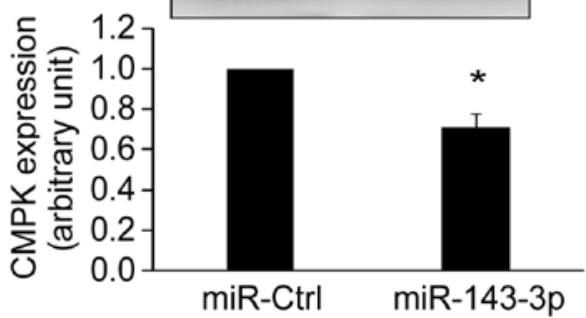

Figure 6. Effect of MALAT1 on miR-143-3p expression. (A and B) Expression of miR-143-3p was detected by qRT-PCR in (A) OVCAR-3 and (B) SK-OV-3 cells transfected with MALAT1-siRNA (siMALAT1) or negative control-siRNA (siNC). Cells without transfection were used as a blank control (Blank). (C) Sequences revealing specific binding sites between MALAT1 and miR-143-3p. MALAT1-wt, wild-type MALAT1; MALAT1-mut, mutated MALAT1. (D) Dual-Luciferase reporter assay. 293T cells were respectively co-transfected with MALAT1-wt/-mut plasmids and miR-143-3p/negative control. (E and F) CMPK protein expression was detected by western blotting. (E) OVCAR-3 and (F) SK-OV-3 cells were transfected with either miR-143-3p or control miRNA (miR-Ctrl). The histogram reveals the quantitative analysis of the gels. The results are presented as the mean $\pm \mathrm{SEM}$. Ctrl, control; ${ }^{*} \mathrm{P}<0.05 ;{ }^{* *} \mathrm{P}<0.01$; $\mathrm{n}=3$ independent experiments. MALAT1, metastasis associated lung adenocarcinoma transcript 1.

released the suppression of $\mathrm{miR}-143-3 \mathrm{p}$ to CMPK inhibition, leading to the progression of OC development.

In conclusion, our findings indicated that MALAT1 is overexpressed in ovarian malignant tumors and influences the survival of patients with OC. Knockdown of MALAT1 affected OC cell behavior. MALAT1 functions as a tumor enhancer by interacting with miR-143-3p and may promote the development OC. Therefore, it is speculated that MALAT1 may serve as a therapeutic target for the treatment of patients with OC. However, an understanding of concrete and extensive mechanisms underlying regulation of MALAT1 in OC needs to be further investigated. 


\section{Acknowledgements}

Not applicable.

\section{Funding}

The present study was supported by grants from the National Natural Science Foundation of China (no. 81272880), the Natural Science Foundation of Shanghai (no. 17ZR1404100), the Science and Technology Commission of Shanghai Municipality (no. 124119b1300), and the Shanghai Municipal Commission of Health and Family Planning (no. 201640287) to GX.

\section{Availability of data and materials}

All data generated or analyzed during this study are included in this published article.

\section{Author's contributions}

QL conducted experiments and performed data analysis, Figure generation and manuscript writing. WG and WR contributed to the collection of clinical samples and pathological diagnosis. WG, LZ and $\mathrm{JZ}$ performed part of the experiments and bioinformatics analyses. GX contributed to the experimental design, data analysis, Figure generation and manuscript writing. All authors read and approved the manuscript and agree to be accountable for all aspects of the research in ensuring that the accuracy or integrity of any part of the work are appropriately investigated and resolved.

\section{Ethics approval and consent to participate}

The present study was approved by the Ethics Committee of Jinshan Hospital.

\section{Consent for publication}

Not applicable.

\section{Competing interests}

The authors declare that they have no competing interests.

\section{References}

1. Siegel RL, Miller KD and Jemal A: Cancer Statistics, 2017. CA Cancer J Clin 67: 7-30, 2017.

2. Auersperg N, Wong AS, Choi KC, Kang SK and Leung PC: Ovarian surface epithelium: Biology, endocrinology, and pathology. Endocr Rev 22: 255-288, 2001.

3. Vaughan S, Coward JI, Bast RC Jr, Berchuck A, Berek JS, Brenton JD, Coukos G, Crum CC, Drapkin R, Etemadmoghadam D, et al: Rethinking ovarian cancer: Recommendations for improving outcomes. Nat Rev Cancer 11: 719-725, 2011.

4. Jayson GC, Kohn EC, Kitchener HC and Ledermann JA: Ovarian cancer. Lancet 384: 1376-1388, 2014.

5. Agarwal R and Kaye SB: Ovarian cancer: Strategies for overcoming resistance to chemotherapy. Nat Rev Cancer 3: 502-516, 2003.

6. Quinn JJ and Chang HY: Unique features of long non-coding RNA biogenesis and function. Nat Rev Genet 17: 47-62, 2016.
7. Wahlestedt C: Targeting long non-coding RNA to therapeutically upregulate gene expression. Nat Rev Drug Discov 12: 433-446, 2013.

8. Batista PJ and Chang HY: Long noncoding RNAs: Cellular address codes in development and disease. Cell 152: 1298-1307, 2013.

9. Schmitt AM and Chang HY: Long noncoding RNAs in cancer pathways. Cancer Cell 29: 452-463, 2016.

10. Hosseini ES, Meryet-Figuiere M, Sabzalipoor H, Kashani HH, Nikzad H and Asemi Z: Dysregulated expression of long noncoding RNAs in gynecologic cancers. Mol Cancer 16: 107, 2017.

11. Gupta RA, Shah N, Wang KC, Kim J, Horlings HM, Wong DJ, Tsai MC, Hung T, Argani P, Rinn JL, et al: Long non-coding RNA HOTAIR reprograms chromatin state to promote cancer metastasis. Nature 464: 1071-1076, 2010.

12. Qu L, Ding J, Chen C, Wu ZJ, Liu B, Gao Y, Chen W, Liu F, Sun W, Li XF, et al: Exosome-transmitted lncARSR promotes sunitinib resistance in renal cancer by acting as a competing endogenous RNA. Cancer Cell 29: 653-668, 2016.

13. Huang FT, Chen WY, Gu ZQ, Zhuang YY, Li CQ, Wang LY, Peng JF, Zhu Z, Luo X, Li YH, et al: The novel long intergenic noncoding RNA UCC promotes colorectal cancer progression by sponging miR-143. Cell Death Dis 8: e2778, 2017.

14. Ji P, Diederichs S, Wang W, Böing S, Metzger R, Schneider PM, Tidow N, Brandt B, Buerger H, Bulk E, et al: MALAT-1, a novel noncoding RNA, and thymosin beta4 predict metastasis and survival in early-stage non-small cell lung cancer. Oncogene 22: 8031-8041, 2003.

15. Tian $\mathrm{X}$ and $\mathrm{Xu}$ G: Clinical value of $\operatorname{lncRNA}$ MALAT1 as a prognostic marker in human cancer: Systematic review and meta-analysis. BMJ Open 5: e008653, 2015.

16. Gutschner T, Hämmerle M, Eissmann M, Hsu J, Kim Y, Hung G, Revenko A, Arun G, Stentrup M, Gross M, et al: The noncoding RNA MALAT1 is a critical regulator of the metastasis phenotype of lung cancer cells. Cancer Res 73: 1180-1189, 2013.

17. Hirata H, Hinoda Y, Shahryari V, Deng G, Nakajima K, Tabatabai ZL, Ishii N and Dahiya R: Long noncoding RNA MALAT1 promotes aggressive renal cell carcinoma through Ezh2 and interacts with miR-205. Cancer Res 75: 1322-1331, 2015.

18. Lai MC, Yang Z, Zhou L, Zhu QQ, Xie HY, Zhang F, Wu LM, Chen LM and Zheng SS: Long non-coding RNA MALAT-1 overexpression predicts tumor recurrence of hepatocellular carcinoma after liver transplantation. Med Oncol 29: 1810-1816, 2012.

19. Fan Y, Shen B, Tan M, Mu X, Qin Y, Zhang F and Liu Y: TGF- $\beta$-induced upregulation of malat1 promotes bladder cancer metastasis by associating with suz12. Clin Cancer Res 20: 1531-1541, 2014.

20. Liu JH, Chen G, Dang YW, Li CJ and Luo DZ: Expression and prognostic significance of lncRNA MALAT1 in pancreatic cancer tissues. Asian Pac J Cancer Prev 15: 2971-2977, 2014.

21. Okugawa Y, Toiyama Y, Hur K, Toden S, Saigusa S, Tanaka K, Inoue Y, Mohri Y, Kusunoki M, Boland CR, et al: Metastasis-associated long non-coding RNA drives gastric cancer development and promotes peritoneal metastasis. Carcinogenesis 35: 2731-2739, 2014.

22. Zheng HT, Shi DB, Wang YW, Li XX, Xu Y, Tripathi P, Gu WL, Cai GX and Cai SJ: High expression of lncRNA MALAT1 suggests a biomarker of poor prognosis in colorectal cancer. Int J Clin Exp Pathol 7: 3174-3181, 2014.

23. Shen L, Chen L, Wang Y, Jiang X, Xia H and Zhuang Z: Long noncoding RNA MALAT1 promotes brain metastasis by inducing epithelial-mesenchymal transition in lung cancer. J Neurooncol 121: 101-108, 2015.

24. Jadaliha M, Zong X, Malakar P, Ray T, Singh DK, Freier SM, Jensen T, Prasanth SG, Karni R, Ray PS, et al: Functional and prognostic significance of long non-coding RNA MALAT1 as a metastasis driver in ER negative lymph node negative breast cancer. Oncotarget 7: 40418-40436, 2016.

25. Chen Q, Su Y, He X, Zhao W, Wu C, Zhang W, Si X, Dong B, Zhao L, Gao Y, et al: Plasma long non-coding RNA MALAT1 is associated with distant metastasis in patients with epithelial ovarian cancer. Oncol Lett 12: 1361-1366, 2016.

26. Jin Y, Feng SJ, Qiu S, Shao N and Zheng JH: LncRNA MALAT1 promotes proliferation and metastasis in epithelial ovarian cancer via the PI3K-AKT pathway. Eur Rev Med Pharmacol Sci 21: 3176-3184, 2017. 
27. Gyorffy B, Lánczky A and Szállási Z: Implementing an online tool for genome-wide validation of survival-associated biomarkers in ovarian-cancer using microarray data from 1287 patients. Endocr Relat Cancer 19: 197-208, 2012.

28. Dweep H and Gretz N: miRWalk2.0: A comprehensive atlas of microRNA-target interactions. Nat Methods 12: 697, 2015.

29. Bayoumi AS, Sayed A, Broskova Z, Teoh JP, Wilson J, Su H, Tang YL and Kim IM: Crosstalk between long noncoding RNAs and microRNAs in health and disease. Int J Mol Sci 17: 356, 2016

30. Zhou D, Zhang L, Sun W, Guan W, Lin Q, Ren W, Zhang J and $\mathrm{Xu}$ G: Cytidine monophosphate kinase is inhibited by the TGF- $\beta$ signalling pathway through the upregulation of miR-130b-3p in human epithelial ovarian cancer. Cell Signal 35: 197-207, 2017.

31. Miao Y, Fan R, Chen L and Qian H: Clinical significance of long non-coding RNA MALAT1 expression in tissue and serum of breast cancer. Ann Clin Lab Sci 46: 418-424, 2016.

32. Li C, Cui Y, Liu LF, Ren WB, Li QQ, Zhou X, Li YL, Li Y, Bai XY and Zu XB: High Expression of Long Noncoding RNA MALAT1 indicates a poor prognosis and promotes clinical progression and metastasis in bladder cancer. Clin Genitourin Cancer 15: 570-576, 2017.

33. Pang EJ, Yang R, Fu XB and Liu YF: Overexpression of long non-coding RNA MALAT1 is correlated with clinical progression and unfavorable prognosis in pancreatic cancer. Tumour Biol 36: 2403-2407, 2015.

34. Wu L, Wang X and Guo Y: Long non-coding RNA MALAT1 is upregulated and involved in cell proliferation, migration and apoptosis in ovarian cancer. Exp Ther Med 13: 3055-3060, 2017.

35. Zou A, Liu R and $\mathrm{Wu} \mathrm{X}$ : Long non-coding RNA MALAT1 is up-regulated in ovarian cancer tissue and promotes SK-OV-3 cell proliferation and invasion. Neoplasma 63: 865-872, 2016.

36. Gutschner T, Hämmerle M and Diederichs S: MALAT1 - A paradigm for long noncoding RNA function in cancer. J Mol Med 91: 791-801, 2013

37. Yoshimoto R, Mayeda A, Yoshida M and Nakagawa S: MALAT1 long non-coding RNA in cancer. Biochim Biophys Acta 1859: 192-199, 2016
38. Salmena L, Poliseno L, Tay Y, Kats L and Pandolfi PP: A ceRNA hypothesis: The Rosetta Stone of a hidden RNA language? Cell 146: 353-358, 2011.

39. Thomson DW and Dinger ME: Endogenous microRNA sponges: Evidence and controversy. Nat Rev Genet 17: 272-283, 2016.

40. Xiao H, Tang K, Liu P, Chen K, Hu J, Zeng J, Xiao W, Yu G, Yao $\mathrm{W}$, Zhou $\mathrm{H}$, et al: LncRNA MALAT1 functions as a competing endogenous RNA to regulate ZEB2 expression by sponging miR-200s in clear cell kidney carcinoma. Oncotarget 6: 38005-38015, 2015.

41. Wang SH, Zhang WJ, Wu XC, Zhang MD, Weng MZ, Zhou D, Wang JD and Quan ZW: Long non-coding RNA Malat1 promotes gallbladder cancer development by acting as a molecular sponge to regulate miR-206. Oncotarget 7: 37857-37867, 2016.

42. Blahna MT and Hata A: Regulation of miRNA biogenesis as an integrated component of growth factor signaling. Curr Opin Cell Biol 25: 233-240, 2013.

43. Desvignes T, Batzel P, Berezikov E, Eilbeck K, Eppig JT, McAndrews MS, Singer A and Postlethwait JH: miRNA nomenclature: A view incorporating genetic origins, biosynthetic pathways, and sequence variants. Trends Genet 31: 613-626, 2015.

44. Li D, Hu J, Song H, Xu H, Wu C, Zhao B, Xie D, Wu T, Zhao J and Fang L: miR-143-3p targeting LIM domain kinase 1 suppresses the progression of triple-negative breast cancer cells. Am J Transl Res 9: 2276-2285, 2017.

45. He Z, Yi J, Liu X, Chen J, Han S, Jin L, Chen L and Song H: MiR-143-3p functions as a tumor suppressor by regulating cell proliferation, invasion and epithelial-mesenchymal transition by targeting QKI-5 in esophageal squamous cell carcinoma. Mol Cancer 15: 51, 2016.

(i)(2) This work is licensed under a Creative Commons Attribution-NonCommercial-NoDerivatives 4.0 International (CC BY-NC-ND 4.0) License. 